\title{
Variations in the dip properties of the low-mass X-ray binary XB 1254-690 observed with XMM-Newton and INTEGRAL
}

\author{
M. Díaz Trigo ${ }^{1}$, A. N. Parmar ${ }^{2}$, L. Boirin ${ }^{3}$, C. Motch ${ }^{3}$, A. Talavera ${ }^{1}$, and S. Balman ${ }^{4}$ \\ 1 XMM-Newton Science Operations Centre, Science Operations Department, ESAC, PO Box 78, 28691 Villanueva de la Cañada, \\ Madrid, Spain \\ e-mail: mdiaz@sciops.esa.int \\ 2 Astrophysics Mission Division, Research and Scientific Support Department of ESA, ESTEC, Postbus 299, 2200 AG Noordwijk, \\ The Netherlands \\ 3 Observatoire Astronomique de Strasbourg, 11 rue de l'Université, 67000 Strasbourg, France \\ 4 Middle East Technical University, Inönü Bulvari, Ankara 06531, Turkey
}

Received 7 May 2008 / Accepted 30 September 2008

ABSTRACT

\begin{abstract}
We have analysed data from five XMM-Newton observations of XB 1254-690, one of them simultaneous with INTEGRAL, to investigate the mechanism responsible for the highly variable dip durations and depths seen from this low-mass X-ray binary. Deep dips were present during two observations, shallow dips during one and no dips were detected during the remaining two observations. At high (1-4 s) time resolution "shallow dips" are seen to include a few very rapid, deep dips whilst the "deep" dips consist of many similar very rapid, deep fluctuations. The folded $V$-band Optical Monitor light curves obtained when the source was undergoing deep, shallow and no detectable dipping exhibit sinusoid-like variations with different amplitudes and phases. We fit EPIC spectra obtained from "persistent" or dip-free intervals with a model consisting of disc-blackbody and thermal Comptonisation components together with Gaussian emission features at 1 and $6.6 \mathrm{keV}$ modified by absorption due to cold and photo-ionised material. None of the spectral parameters appears to be strongly correlated with the dip depth except for the temperature of the disc blackbody which is coolest $(k T \sim 1.8 \mathrm{keV})$ when deep dips are present and warmest $(k T \sim 2.1 \mathrm{keV})$ when no dips are detectable. We propose that the changes in both disc temperature and optical modulation could be explained by the presence of a tilted accretion disc in the system. We provide a revised estimate of the orbital period of $0.16388875 \pm 0.00000017$ day.
\end{abstract}

Key words. X-rays: binaries - X-rays: individuals: XB 1254-690 - accretion, accretion disks

\section{Introduction}

Around 10 galactic low-mass X-ray binaries (LMXBs) exhibit periodic dips in their X-ray intensity. The dips are believed to be caused by periodic obscuration of the central X-ray source by structure located in the outer regions of a disc (White \& Swank 1982). The depth, duration and spectral evolution of the dips vary from source to source and often from cycle to cycle. The 1-10 keV spectra of most of the dip sources become harder during dipping. However, these changes are inconsistent with a simple increase in photo-electric absorption by cool material, as an excess of low-energy photons is usually present. Narrow absorption features from highly ionised $\mathrm{Fe}$ and other metals have been observed from a number of dipping LMXBs and microquasars (e.g., Ueda et al. 1998; Sidoli et al. 2001; Lee et al. 2002). The important role that photo-ionised plasmas play in LMXBs was recognised by Boirin et al. (2005) and Díaz Trigo et al. (2006) who were able to model the changes in both the narrow X-ray absorption features and the continuum during the dips from all the bright dipping LMXB observed by XMM-Newton by an increase in the column density and a decrease in the amount of ionisation of a photo-ionised absorbing plasma. Since dipping sources are normal LMXBs viewed from close to the orbital plane, this implies that photo-ionised plasmas are common features of LMXBs. Outside of the dips, the properties of the absorbers do not vary strongly with orbital phase suggesting that the ionised plasma has a cylindrical geometry with a maximum column density close to the plane of the accretion disc.

Dipping activity from the LMXB XB 1254-690 was discovered during EXOSAT observations in 1984 when the source exhibited irregular reductions in X-ray intensity that repeated every $3.88 \pm 0.15 \mathrm{~h}$ (Courvoisier et al. 1986). In a 1984 August observation, five deep dips were observed with a mean duration of $\sim 0.8 \mathrm{~h}$ and a reduction in $1-10 \mathrm{keV}$ intensity of $\sim 95 \%$. $V$-band observations of the 19th magnitude companion identified by Griffiths et al. (1978) revealed an optical modulation with minima occurring $\sim 0.2$ cycles after the X-ray dips (Motch et al. 1987). The optical modulation has a period of $3.9334 \pm 0.0002 \mathrm{~h}$, consistent with the mean X-ray dip recurrence interval, and can be modelled as resulting primarily from viewing different aspects of the X-ray heated atmosphere of the companion star with a small contribution from the bulge where the accretion stream impacts the outer disc (Motch et al. 1987). This indicates that the accretion disc does not entirely shadow the companion. The presence of dips and the lack of X-ray eclipses provides a constraint on the inclination angle, $i$, of the source of between $65^{\circ}$ and $73^{\circ}$ (Courvoisier et al. 1986; Motch et al. 1987).

Deep dips were present during a Ginga observation of XB 1254-690 in 1990 (Uno et al. 1997), but were not detected during Rossi X-ray Timing Explorer (RXTE) observations in 1997 (Smale \& Wachter 1999) and BeppoS AX observations in 1998 (Iaria et al. 2001). Optical observations in 1997 revealed 
Table 1. XMM-Newton observations of XB 1254-690.

\begin{tabular}{|c|c|c|c|c|c|c|c|c|}
\hline \multirow{2}{*}{$\begin{array}{l}\text { Obs. } \\
\text { Num. }\end{array}$} & \multirow{2}{*}{$\begin{array}{l}\text { Observation } \\
\text { ID }\end{array}$} & \multicolumn{3}{|c|}{ Observation Times (UTC) } & \multirow{2}{*}{$\begin{array}{l}\text { Dip. } \\
\text { Depth }\end{array}$} & \multirow{2}{*}{$\begin{array}{c}T \\
(\mathrm{ks})\end{array}$} & \multirow{2}{*}{$\underset{\left(\mathrm{s}^{-1}\right)}{C}$} & \multirow[t]{2}{*}{$H R$} \\
\hline & & $\begin{array}{r}\text { Start } \\
\text { (year month day) }\end{array}$ & (h:mn) & $\begin{array}{l}\text { End } \\
(\mathrm{h}: \mathrm{mn})\end{array}$ & & & & \\
\hline 1 & 0060740101 & 2001 January 22 & $15: 49$ & $20: 03$ & Deep & 15.0 & $170.8 \pm 0.2$ & $0.691 \pm 0.001$ \\
\hline 2 & 0060740901 & 2002 February 7 & $17: 32$ & $25: 08$ & Undetected & 26.8 & $178.2 \pm 0.2$ & $0.770 \pm 0.001$ \\
\hline 3 & 0405510301 & 2006 September 12 & $16: 15$ & 08:01 & Undetected & 56.0 & $192.5 \pm 0.2$ & $0.7665 \pm 0.0006$ \\
\hline 4 & 0405510401 & 2007 January 14 & $01: 13$ & $18: 20$ & Deep & 60.8 & $178.3 \pm 0.2$ & $0.7044 \pm 0.0005$ \\
\hline 5 & 0405510501 & 2007 March 9 & 04:03 & $20: 44$ & Shallow & 59.2 & $183.0 \pm 0.2$ & $0.7267 \pm 0.0007$ \\
\hline
\end{tabular}

Results from the first two observations were reported in Boirin \& Parmar (2003) and Díaz Trigo et al. (2006). $T$ is the total EPIC pn exposure time, $C$ is the pn $0.6-10 \mathrm{keV}$ persistent emission background subtracted count rate and $H R$ the hardness ratio (counts in the $2-10 \mathrm{keV}$ energy range divided by those between 0.6-2 keV) for the dip-free intervals. Obs. 4 was simultaneous with INTEGRAL. In all cases the EPIC pn thin filter was used. "Deep" is a reduction of $\sim 10-90 \%$ in the $0.6-10 \mathrm{keV} \mathrm{X}$-ray flux within $1 \mathrm{~h}$ around the dip centre compared to the flux within $1 \mathrm{~h}$ measured $2 \mathrm{~h}$ away from the dip centre, "shallow" is a reduction of $\sim 5-10 \%$ and "undetected" implies a reduction of $\lesssim 5 \%$.

that the mean $V$ magnitude was unchanged, but that the amplitude of the optical variability had declined by $\Delta V \sim 0.1 \mathrm{mag}$ (Smale \& Wachter 1999). This may be explained if the vertical structure at the outer regions of the disc responsible for the dips decreased in angular size from $17^{\circ}-25^{\circ}$ to $<10^{\circ}$ (Smale \& Wachter 1999). The dips had re-appeared during XMM-Newton and RXTE observations in 2001 January and May, but were again not present during observations in 2001 December (Smale et al. 2002), 2002 February (Boirin \& Parmar 2003) and 2003 October (Iaria et al. 2007). Deep dipping had returned in an RXTE observation in 2004 May (Barnes et al. 2007). While all the well studied LMXB dippers show variability from cycle to cycle, the large variations in dip depth observed from XB 1254-690 are unusual.

Here, we report the results of three XMM-Newton observations of XB 1254-690 in 2006 and 2007 as well as a re-analysis of the two earlier XMM-Newton observations reported in Boirin \& Parmar (2003) and Díaz Trigo et al. (2006). The new observations include results from the Optical Monitor which was not operated in the early observations. The second of the new observations was simultaneous with an INTEGRAL observation which is used to extend the analysis to higher energies. The goal of this work is to investigate and explain the mechanism presumed responsible for the large changes in dip depth.

\section{Observations}

\subsection{XMM-Newton observations}

The XMM-Newton Observatory (Jansen et al. 2001) includes three $1500 \mathrm{~cm}^{2}$ X-ray telescopes each with an EPIC (0.1-15 keV) at the focus. Two of the EPIC imaging spectrometers use MOS CCDs (Turner et al. 2001) and one uses pn CCDs (Strüder et al. 2001). The RGSs $(0.35-2.5 \mathrm{keV}$, Den Herder et al. 2001) are located behind two of the telescopes. In addition, there is a co-aligned $30 \mathrm{~cm}$ diameter Optical/UV Monitor telescope (OM, Mason et al. 2001), simultaneously providing coverage with the X-ray instruments. Data products were reduced using the Science Analysis Software (SAS) version 7.1. Since the EPIC pn is more sensitive to the presence of Fe lines than the MOS CCDs, with an effective area a factor $\sim 5$ higher at $7 \mathrm{keV}$, we concentrate here on the analysis of pn data from EPIC. RGS data from both gratings in first and second order were also used, as were OM data from the three available XMM-Newton observations.

Table 1 is a summary of the XMM-Newton observations. We used the EPIC pn in Timing Mode. In this mode only one
CCD chip is operated and the data are collapsed into a onedimensional row (4.4) and read out at high speed, the second dimension being replaced by timing information. This allows a time resolution of $30 \mu \mathrm{s}$, and photon pile-up occurs only for count rates $>800$ counts $\mathrm{s}^{-1}$, well in excess of the observed count rates (see Fig. 1). Only single and double Timing Mode events (patterns 0 to 4 ) were selected and source events were extracted from a 53" wide column centred on the source position. Background events were obtained from a column of the same width, but positioned away from the source. Ancillary response files were generated using the SAS task arfgen. Response matrices were generated using the SAS task rmfgen. The SAS task rgsproc was used to produce calibrated RGS event lists, spectra, and response matrices. We chose the option keepcool $=$ no to discard single columns that give signals a few percent below the values expected from their immediate neighbours. Such columns are likely to be important when studying weak absorption features in spectra with high statistics. The OM was operated in Image + Fast Mode in Obs. 3-5. The $V$ filter, very close to the standard Johnson $V$ band, was used. In this mode the instrument produces images of the entire $17^{\prime} \times 17^{\prime}$ FOV with a time resolution between 800 and $5000 \mathrm{~s}$ and event lists with a time resolution of $0.5 \mathrm{~s}$ from a selected $11^{\prime \prime} \times 11^{\prime \prime}$ region. The SAS task omfchain was used to extract light curves of XB 1254-690 from the high time resolution Fast Mode data. We used a time sampling of $100 \mathrm{~s}$ in the curve extraction to improve the signalto-noise ratio. We corrected the OM Fast Window light curves and EPIC pn event times to the solar system barycentre using the SAS task barycen.

\subsection{INTEGRAL observation}

The INTEGRAL (Winkler et al. 2003) payload consists of two main gamma-ray instruments, one of which is optimized for $15 \mathrm{keV}$ to $10 \mathrm{MeV}$ high-resolution imaging (IBIS; Ubertini et al. 2003). IBIS provides an angular resolution of $12^{\prime}$ fullwidth at half-maximum ( $F W H M)$ and an energy resolution, $E / \triangle E$, of $\sim 12 F W H M$ at $100 \mathrm{keV}$. The extremely broad energy range of IBIS is covered by two separate detector arrays, ISGRI (15-1000 keV; Lebrun et al. 2003) and PICsIT (0.175-10 MeV; Labanti et al. 2003). The payload is complemented by an X-ray monitor (JEM-X; 3-35 keV; Lund et al. 2003). JEM-X has a fully coded FOV of 4.8 diameter and an angular resolution of $3^{\prime}$ FWHM.

An INTEGRAL observation of the region of sky containing XB 1254-690 was performed between 2007 January 14 02:02 and 2007 January 15 05:47 UTC, for a total time of $74 \mathrm{ks}$, 

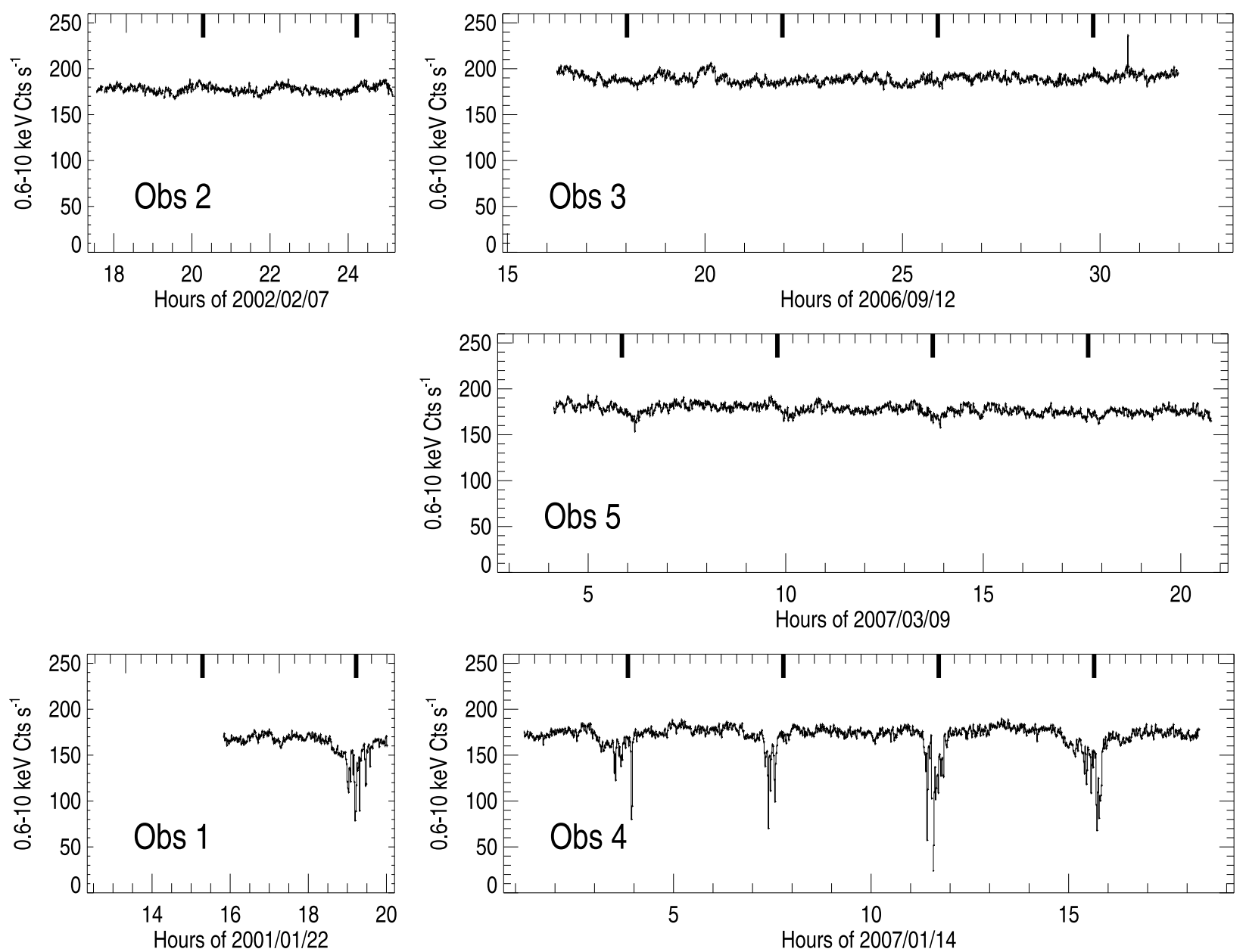

Fig. 1. 0.6-10 keV EPIC pn background subtracted light curves for each XB 1254-690 observation with a binning of $120 \mathrm{~s}$. The panels have been arranged so that the observations not showing dips (Obs. 2 and 3) are shown at the top, the observation showing shallow dips (Obs. 5) in the middle and the observations showing deep dips (Obs. 1 and 4) at the bottom. The axes scales are the same for all observations. The thick vertical tick marks indicate the expected dip centres using the period of optical variations given in Motch et al. (1987) and using the deep dips in Obs. 4 to define the dip phase (see text). A burst is visible in Obs. 3 .

coinciding with XMM-Newton Obs. 4 . The standard $5 \times 5$ dither patterns of pointings centred on the target position were performed. Data from the observation were processed using the Offline Scientific Analysis (OSA) version 7.0. In this paper we use data from ISGRI and JEM-X, both of which use coded masks to provide imaging information. This means that photons from a source within the FOV are distributed over the detector area in a pattern determined by the position of the source in the FOV. Source positions and intensities are determined by matching the observed distribution of counts with those produced by the mask modulation.

\subsection{X-ray light curves}

Figure 1 shows $0.6-10 \mathrm{keV}$ EPIC pn light curves of all five XMM-Newton observations of XB 1254-690 with a binning of $120 \mathrm{~s}$. The plots have been grouped so that the upper panels show the two observations where dipping was not detected (Obs. 2 and 3), the middle panels the observation where shallow dipping was detected (Obs. 5), and the lower panels the two observations with deep dipping (Obs. 1 and 4). "Deep" is defined as a reduction of the $0.6-10 \mathrm{keV}$ flux of $\sim 10-90 \%$ when integrated over $1 \mathrm{~h}$ around the dip centre compared to the flux within $1 \mathrm{~h}$ measured
$2 \mathrm{~h}$ away from the dip centre, "shallow" is a similar reduction of $\sim 5-10 \%$ and "undetected" implies a reduction of $\lesssim 5 \%$. The thick vertical tick marks indicate the expected dips times derived using the reference epoch of JD 2454114.65502 and a folding period of $0.163890 \pm 0.000009$ day (Motch et al. 1987). The reference epoch corresponds to the centre of the first X-ray dip in Obs. 4 and was determined by folding the $0.6-10 \mathrm{keV}$ EPIC pn light curve of Obs. 4 with the source period and fitting the resultant deep dip with a negative Gaussian function.

The dip shape and depth vary from dip to dip during Obs. 1 and 4. However, the dips seem to follow a regular pattern. An EPIC pn 0.6-10 keV light curve and hardness ratio (counts in the $2-10 \mathrm{keV}$ energy range divided by those between $0.6-2 \mathrm{keV}$ ) plot of a deep dip with a time resolution of $4 \mathrm{~s}$ are shown in the upper left panels of Fig. 2. The lower left panels show part of the same dip with a time resolution of $1 \mathrm{~s}$. Examination of the upper panels shows that the intensity first decreases slightly ("shoulder") while the hardness ratio remains more or less unchanged, and then at some point both the intensity and the hardness ratio become highly variable on a wide range of timescales. The deepest segments of the dips are associated with the strongest hardening. Light curves and hardness ratio plots from the shallow dips seen in Obs. 5 are shown in the right panels. When plotted at the 

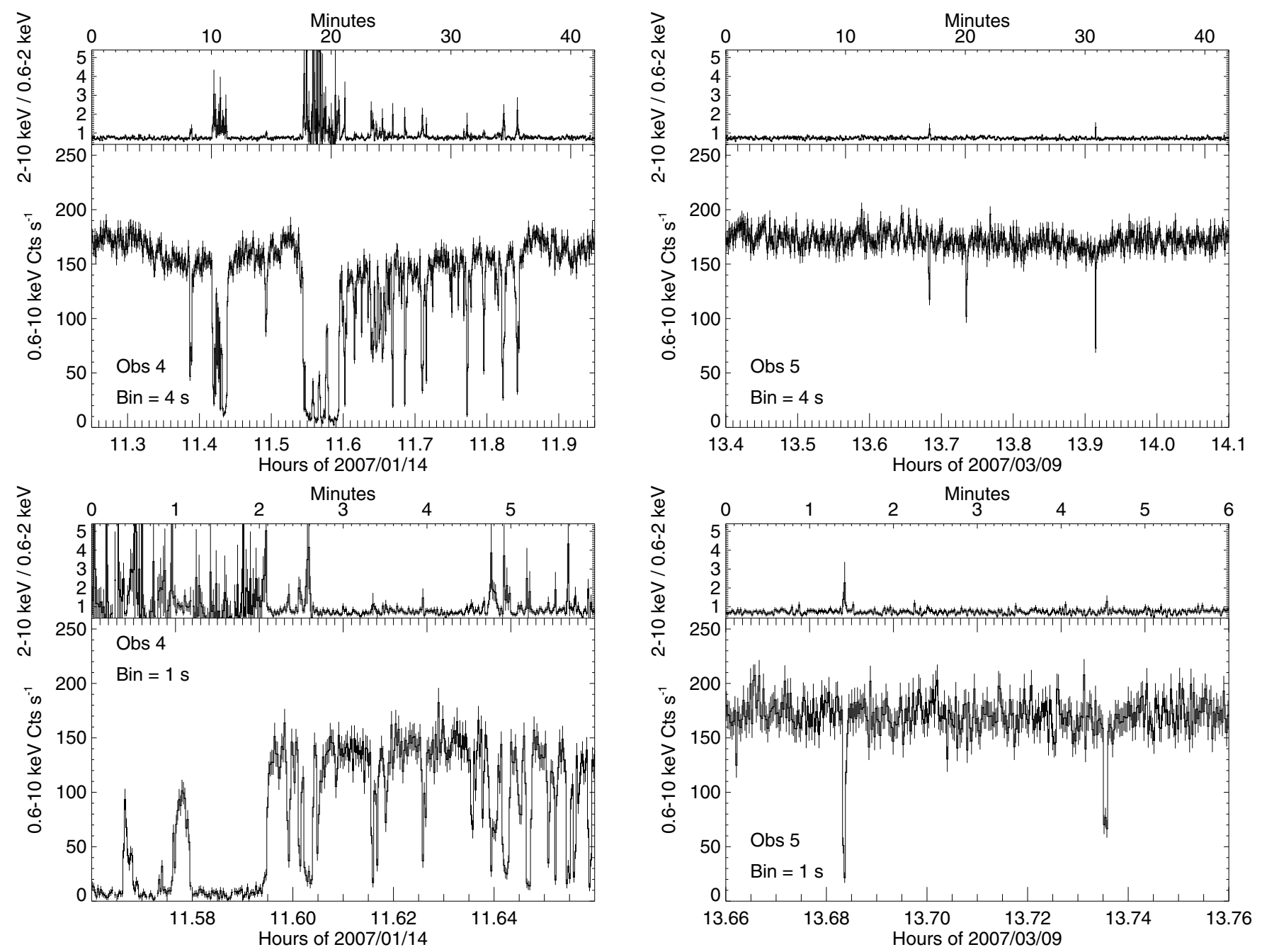

Fig. 2. EPIC pn 0.6-10 keV light curves and hardness ratios for XB 1254-690, with a binning of $4 \mathrm{~s}$ (upper panels) and $1 \mathrm{~s}$ (lower panels), showing many, rapid, and deep intensity fluctuations during a deep dip (left). The "shoulder" (see definition in Sect. 2.3) is visible between 11.3 and $11.4 \mathrm{~h}$ in the upper left panel. In contrast, the shallow dip (right), when plotted at high time resolution, is seen to consist of a smaller number of similar rapid deep intensity fluctuations. The hardness ratio is counts in the $2-10 \mathrm{keV}$ energy range divided by those between $0.6-2 \mathrm{keV}$.

time resolution of Fig. 1 (120 s) the shallow dips appear similar to the "shoulders" of the deep dips in the rebinned light curve. However, at the higher 1 or 4 s time resolutions of Fig. 2 it is evident that the shallow dip also includes few very short duration deep dips with depths and hardness ratios similar to the deep dips seen during Obs. 1 and 4 . We examined the hardness ratio as a function of the $0.6-10 \mathrm{keV}$ count rate for the dip-free intervals of all the observations (Fig. 3). The hardness ratio increases from the observations showing deep dipping (Obs. 1 and 4) to the one showing shallow dipping (Obs. 5) and is largest for the observations where dipping behaviour is absent (Obs. 2 and 3). The average count rate increased from the 2000-2001 observations (Obs. 1 and 2) to the 2006-2007 ones (Obs. 3 to 5). A higher count rate during observations where dipping is absent compared to observations where dipping is present is observed in both epochs.

We note that an X-ray burst is observed in 2006 September 13 at 6.71 h during Obs. 3 (see Fig. 4).

\subsection{Folded lightcurves}

We extracted light curves from the $V$ filter OM Fast Window exposures performed in Obs. 3-5, with a time bin of $100 \mathrm{~s}$. The
OM was not operated during Obs. 1 and 2. The background region was carefully defined to avoid contamination by field stars located close to the optical counterpart of XB 1254-690, which were present in the OM Fast Window region. During some exposures in Obs. 3 the optical counterpart was located close to the edge, or even outside, of the Fast Window region due to spacecraft pointing drift. Based on the known OM point spread function, we rejected exposures where $>50 \%$ of the source flux was outside the Fast Window region as it was difficult to reliably reconstruct the total count rates. The light curves show a sinusoid-like modulation consistent with the period of the binary. However, at a similar time resolution as the X-ray light curve in Fig. 1, the uncertainties on the OM lightcurves are too large to reveal details of the modulation. Thus, we resampled the light curves at $1416 \mathrm{~s}$ (one tenth of the binary period) and folded the light curves using the ephemeris presented earlier and examined each observation separately ${ }^{1}$.

\footnotetext{
1 We note that SAS calculates the errors for the OM light curves based on a Bernouilli distribution rather than a Poisson distribution (Kuin \& Rosen 2008). Therefore, the Gaussian error propagation used for the folding of the curves and the $\chi^{2}$ statistic may be not appropriate.
} 


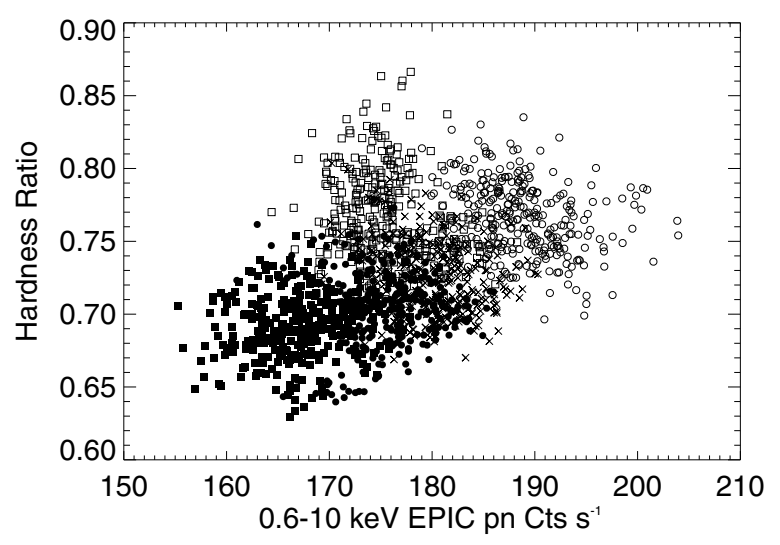

Fig. 3. Hardness ratio (2-10 keV/0.6-2 keV counts) versus $0.6-10 \mathrm{keV}$ EPIC pn count rate during dip-free intervals. Data from Obs. 1 to 5 are shown as filled squares, open squares, open circles, filled circles and crosses, respectively. An increase of the hardness ratio from the observations showing deep dipping (filled symbols) to the observations where dipping is absent (open symbols) is visible.

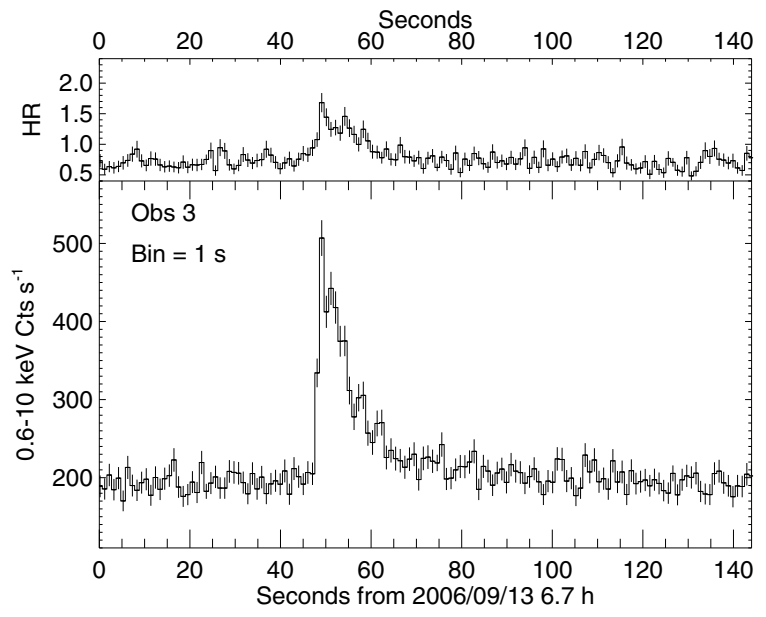

Fig. 4. The 0.6-10 keV EPIC pn light curve and hardness ratio (counts in the $2-10 \mathrm{keV}$ energy range divided by those between $0.6-2 \mathrm{keV}$ ) for XB 1254-690 Obs. 3 during the burst with a binning of $1 \mathrm{~s}$.

The folded light curves reveal differences in shape and magnitude between the three observations. Figure 5 shows $V$ filter OM (lower panels) and simultaneous 0.6-10 keV EPIC pn (upper panels) light curves of XB 1254-690 folded on the refined orbital period determined in Sect. 2.6, where $\phi=0$ corresponds to optical minimum. We used the same ephemeris to fold all 3 observations. The Obs. 3 OM light curve shows evidence for a secondary minimum at $\phi \sim 0.5$ which may also be present in Obs. 4 as an apparent flattening of the curve around this phase, but is clearly absent in Obs. 5. The average EPIC pn count rate decreased from Obs. 3 to Obs. 5. Finally, the phases of both the optical and X-ray modulations changed between the observations. In X-rays, we observe that the shallow dips in Obs. 5 appear $\sim 0.1$ later in phase compared to the deep dips in Obs. 4. This difference is unlikely to be due to uncertainties in the ephemeris and the period propagation since the accumulated uncertainty from Obs. 4 to Obs. 5 is estimated to be $\phi<0.0005$ (see Sect. 2.6). Similarly, while the optical minimum falls near $\phi=0$ for both Obs. 3 and 5, it occurs later at $\phi=0.1$ for Obs. 4 , when deep dips are present.
Table 2. Best-fits to the folded OM light curves for Obs. 3-5 using the $M+A^{*} \sin \left(2 \pi\left(c * \phi+0.25-\phi_{0}\right)\right)$ model.

\begin{tabular}{lccc}
\hline \hline Obs. & 3 & 5 & 4 \\
\hline Param. & & & \\
$M$ & $18.99 \pm 0.03$ & $19.12 \pm 0.03$ & $19.07 \pm 0.03$ \\
$A$ & $0.15 \pm 0.04$ & $0.25 \pm 0.04$ & $0.17 \pm 0.04$ \\
$\phi_{0}$ & $0.99 \pm 0.04$ & $0.93 \pm 0.03$ & $0.11 \pm 0.03$ \\
\hline$\chi_{v}^{2}$ (d.o.f.) & $0.84(13)$ & $0.29(13)$ & $0.26(13)$ \\
\hline
\end{tabular}

$M, A, \phi$, and $\phi_{0}$ are the average optical magnitude, amplitude, phase and phase at the optical minimum, respectively. The coefficient $c$ has been fixed to 1 , since the curves are folded on the refined orbital period determined in Sect. 2.6.

We modelled the OM folded light curves with a sinusoid with the period fixed at the orbital value. This model fits well the folded light curves of Obs. 4 and 5. The fit quality for Obs. 3 is slightly worse than the others due to the possible presence of a secondary minimum at $\phi \sim 0.5$ which is not modelled. The results of the fits are shown in the lower panels of Fig. 5 and given in Table 2. Finally, we subtracted the OM light curve of Obs. 4 from Obs. 3 and 5 (see Fig. 6). An excess is observed at $\phi \sim 0.8$ in both subtracted light curves, indicating additional optical emission at this phase in Obs. 4, when deep dipping was present, compared to Obs. 3 and 5.

\subsection{Dip recurrence interval}

The most accurate orbital ephemeris for XB 1254-690 is that of Motch et al. (1987) who derived a period of $0.163890 \pm 0.000009$ day with a time of optical minimum of JD $2445735.693 \pm 0.004$. This ephemeris is not accurate enough to maintain phase between the two XMM-Newton observations where deep dips were detected in 2001 January and 2007 January as the accumulated uncertainty on the predicted time of $\pm 2.9 \mathrm{~h}$ is close to a complete orbital cycle. Recently, Barnes et al. (2007) updated the orbital ephemeris based on an RXTE Proportional Counter Array observation on 2004 May 26 that includes a deep dip. They estimate a new value of JD 2453151.509 (3) for the optical minimum $(\phi=0)$ assuming that the centre of the X-ray dip occurred at $\phi=0.84$. The centre of the first deep dip observed by XMM-Newton in 2007 January 14 (Obs. 4) occurred at 3.72 h UTC (see Sect. 2.3). Using the XMM-Newton dip to define the dip phases and using the Motch et al. (1987) period gives a predicted time of $3.30 \mathrm{~h}$ for the dip observed by RXTE, only $0.23 \mathrm{~h}$ before the dip time given by Barnes et al. (2007). Given that the predicted uncertainty in the dip times is $\pm 1.3 \mathrm{~h}$, this is an unusually close prediction. Similarly, the predicted time for the dip observed by XMM-Newton in the 2001 January 22 observation (Obs. 1) is $19.10 \mathrm{~h}$, only $0.12 \mathrm{~h}$ before the time given by Boirin \& Parmar (2003), whereas the uncertainty in the Motch et al. (1987) period determination corresponds to $\pm 2.9 \mathrm{~h}$. It is unlikely that both of these close predictions are coincidences and instead we suggest that the uncertainty on the Motch et al. (1987) ephemeris has been overestimated by a factor of $\sim 5$. This would imply a dip recurrence period of $0.163890 \pm 0.000002$ day. 


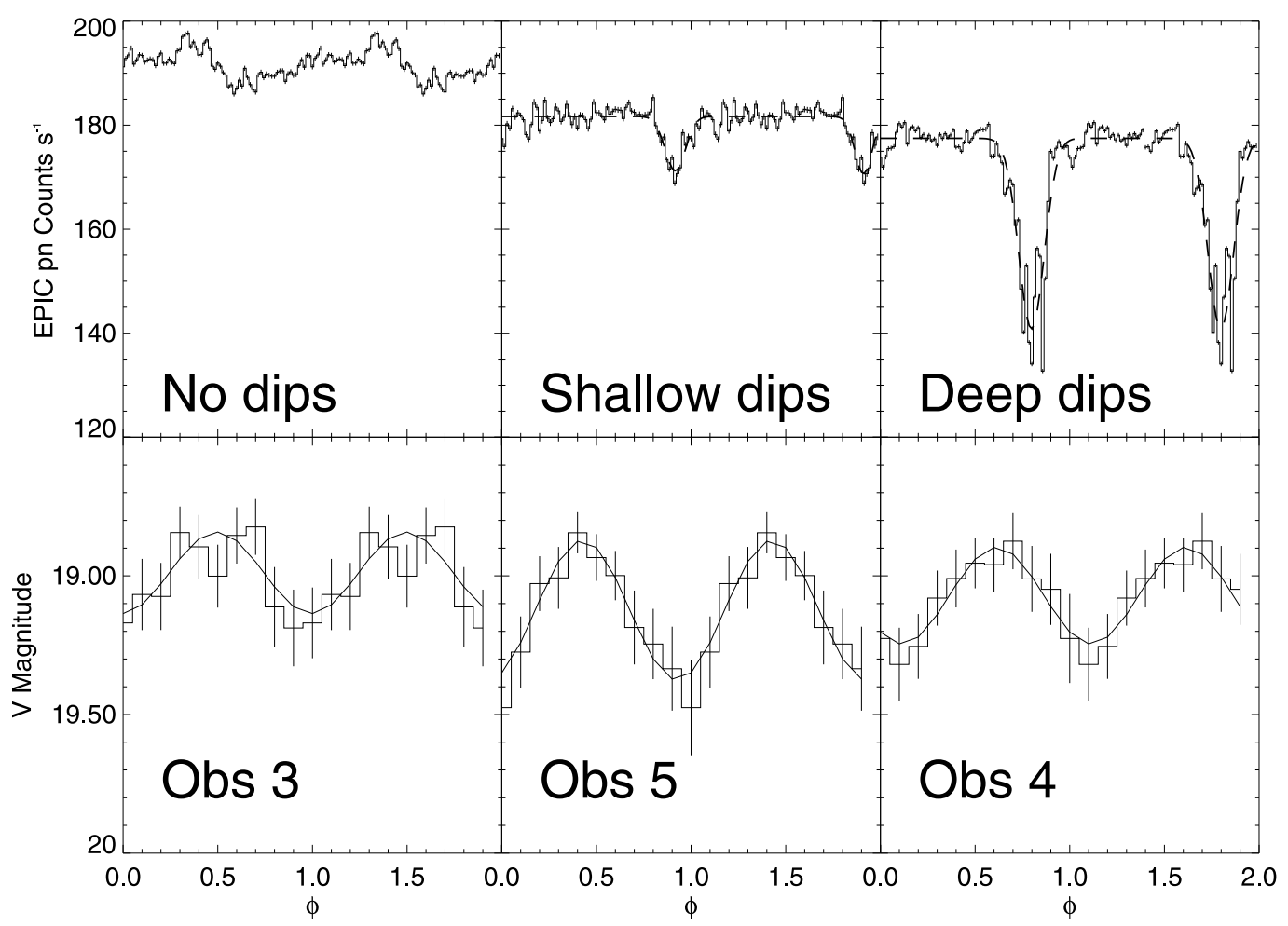

Fig. 5. 0.6-10 keV EPIC pn (upper panels) and $V$ filter OM (lower panels) XB 1254-690 light curves folded at the refined orbital period determined in Sect. 2.6 when no dips, shallow dips and deep dips were present. The reference epoch for phase is the same for all the plots and $\phi=0.0$ corresponds to optical minimum. The reference phase for the optical minimum has been calculated taking into account the dip reference phase calculated in Sect. 2.3 and assuming that the dip centre occurs at $\phi=0.8$. The binning is $1416 \mathrm{~s}$ and $200 \mathrm{~s}$ for the OM and EPIC pn light curves, respectively. The solid lines in the lower panels show the fit of Table 2.

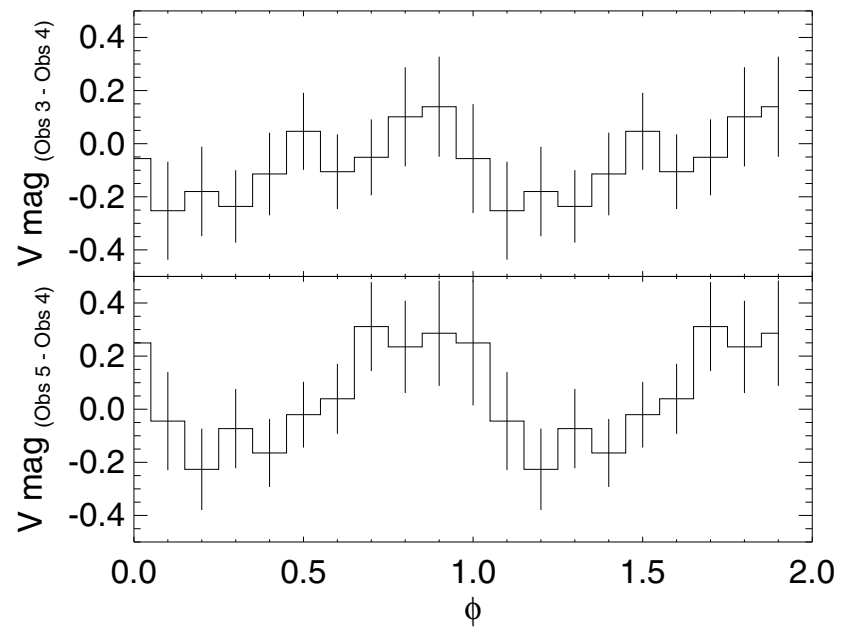

Fig. 6. Differences between the OM light curves shown in Fig. 5 (lower panels). The upper panel shows the difference between the light curves of Obs. 3 and 4 and the lower panel between Obs. 5 and 4. An excess of optical emission is seen at $\phi \sim 0.8$ in Obs. 4, when deep dipping was taking place.

\subsection{Light curves from other $X$-ray missions}

We have shown that at the 1 or $4 \mathrm{~s}$ time resolution of Fig. 2, shallow dips include a few very short duration deep dips each with depths and hardness ratios similar to the longer duration dips seen during Obs. 1 and 4 (see Sect. 2.3). In order to investigate the dip variability further, we extracted from the High Energy Astrophysics Science Archive Research Center (HEASARC) light curves from previous XB 1254-690 X-ray observations with the maximum time resolution available. We then searched for the signature of shallow dips that may have been missed due to the use of too coarse binning. Our goal was to determine the minimum time between the disappearance and re-appearance of deep and shallow dips and thus to see if there is a characteristic time scale for these changes which might provide a clue as to the underlying mechanism. An overview of the dip states seen in the different X-ray observations of XB 1254-690, following the definition of Table 1 , is given in Table 3.

The variations in dip depth could result from some irregular process and occur on a range of time scales. Alternatively, there could be an underlying regular mechanism such as disc precession which could give rise to a characteristic time scale for the variations. In order to investigate possible characteristic time scales we examined all available X-ray light curves. The longest uninterrupted interval with deep dipping activity occurs within the $19 \mathrm{~h}$ duration EXOSAT observation on 6-7 August 1984, when five consecutive deep dips are observed. Similarly, the longest interval without dipping activity is observed in the RXTE pointed observation on 6-7 December 2001, when dipping was absent during 6 consecutive cycles, or $19 \mathrm{~h}$. In XMM-Newton Obs. 5 four consecutive shallow dips are observed over $17 \mathrm{~h}$. These measurements give a lower limit of $\gtrsim 20 \mathrm{~h}$ for any characteristic time scale of a given dipping state. In contrast, a transition from deep dipping to non-dipping is observed during the EXOSAT observation of 5 February 1984 in only one orbital cycle, or $4 \mathrm{~h}$. The long $60 \mathrm{~h}$ (but non-continuous) RXTE pointed observation on 9-12 May 2001 provides additional constraints. Here we find a complex sequence of dip behaviour consisting of a deep dip, a gap of 5 cycles (due to incomplete coverage), two non-dip episodes, a gap of one orbital cycle, 
a shallow dip, a gap of another orbital cycle, a dip that could be shallow or deep, a gap of 2 cycles, and 2 further dips that are not fully covered and could be deep or shallow. Thus, if any of the last three dip episodes is deep, this would imply a re-appearance of deep dipping within $\sim 60 \mathrm{~h}$. Given that a sharp variation in dip properties was observed within a single $4 \mathrm{~h}$ orbital cycle, that the dip patterns can be unchanged for $\sim 20 \mathrm{~h}$ intervals and that a $60 \mathrm{~h}$ observation revealed the whole range of dip depths suggests that if there is a characteristic timescale for dip depth variability it is around $60 \mathrm{~h}$.

We also examined the XB 1254-690 RXTE All-Sky Monitor (ASM) 1.5-12 keV light curves spanning 12 years and with a time resolution of $96 \mathrm{~s}$ to search for any characteristic timescale for the appearance and disappearance of dips. We corrected the ASM light curves to the solar system barycentre using the FTOOL task faxbary and folded the light curves into segments of between 4 and 200 orbital periods duration using the ephemeris and period from Sect. 2.3, but clear dipping activity was not detected due to the low count rates.

Next, we investigated whether the orbital period of the source can be refined from the ASM light curves. We first calculated the Lomb-Scargle periodogram (Scargle 1982) which revealed a clear peak at the optical period. Its probability of being due to a random fluctuation is $\sim 4 \times 10^{-6}$ implying a clear detection of the X-ray orbital modulation in the ASM data. The ASM folded light curve shows a clear dip at the expected phase $(\phi \sim 0.8$, see Fig. 7). The reduction in intensity compared to the persistent level of $2.3 \mathrm{~s}^{-1}$ is $10 \%$. This low value is consistent with averaging intervals with deep, shallow and no dipping activity. The dip is preceded by a "shoulder" with a reduced intensity of $\sim 5 \%$ with respect to the persistent level at $\phi \sim 0.7$. Such "shoulders" are clearly seen in the XMM-Newton light curves (see Sect. 2.3) and indicate the high sensitivity that can be achieved using ASM light curves spanning a long time period.

Although the dips may not be observed during all orbital cycles, they do provide a sharp fiducial mark which can be used to determine an improved ephemeris. We used the $\chi^{2}$ test on folded data (Leahy et al. 1983) which is sensitive to the total power, not only the fundamental, to better constrain the value of orbital period. The optical period is well detected with a $\chi^{2}$ of 85.10 for 21 degrees of freedom (d.o.f.). We performed Monte Carlo simulations in order to estimate the uncertainty on the best period. The actual ASM time series folded at the test frequency provided a mean pattern from which a fake light curve was created by adding Gaussian noise with $\sigma$ proportional to the error associated with each actual measurement. From a total of 1000 runs we derived the distribution of the frequency at which the maximum $\chi^{2}$ of the periodogram occurs. We find a best period of $0.16388875 \pm 0.00000017 \mathrm{~d}$, consistent with that derived by Motch et al. (1987) from optical data and with the one determined in Sect. 2.5, improving by a factor 50 in accuracy. We provide a revised ephemeris of JD 2454114.6877975 for the optical minimum. This ephemeris has been derived taking into account the dip reference phase calculated in Sect. 2.3 and assuming that the dip centre occurs at $\phi=0.8$, and is the same used to fold the ASM light curve shown in Fig. 7.

Finally, we studied the long-term dipping behaviour of XB 1254-690 with the available ASM data. Fig. 8 shows the count rate during dipping $(\phi=0.6-1.0)$ and persistent phases ( $\phi=0.0-0.6)$ (left panel) and the ratio of these quantities (right panel) along the 12 years of ASM data. In general, the periods of low persistent count rate $\left(\$ 2.2 \mathrm{cts} \mathrm{s}^{-1}\right)$ show a "Dip Ratio" significantly below 1 , indicating strong dipping activity. In contrast, the pointed observations showing deep dipping activity

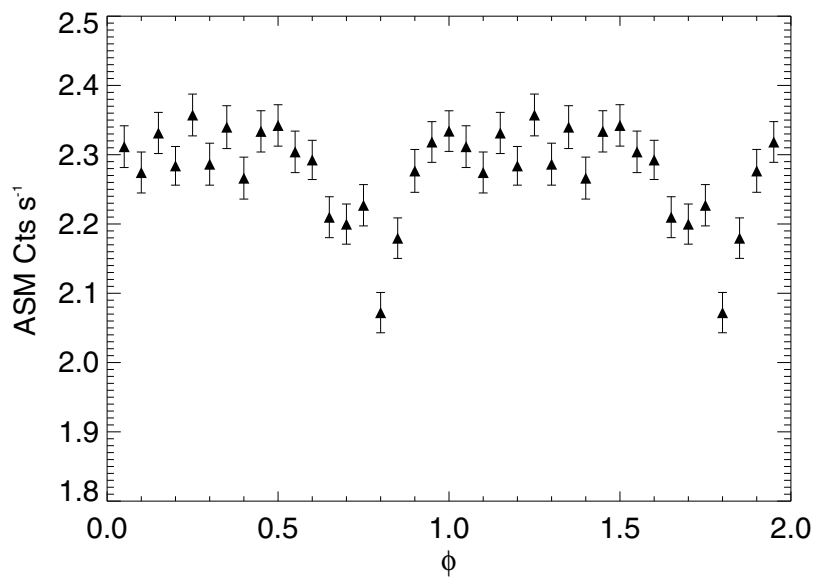

Fig. 7. ASM 1.5-12 keV background subtracted light curve for XB 1254-690 folded at the refined orbital period determined in Sect. 2.6, showing the clear detection of dipping at $\phi \sim 0.8$. We have used the same reference phase as in Fig. 5.

(see Table 3) are distributed along the whole time range. Given the small amount of coverage of pointed observations, we cannot exclude a relation between the detection of deep dipping in pointed observations and the ASM count rate.

\subsection{X-ray spectra}

For the observations showing deep and shallow dipping, we first selected intervals of "persistent" or dip-free emission. Spectra were accumulated corresponding to these intervals and for the entire set of dip-free observations. We rebinned the EPIC pn spectra to over-sample the $F W H M$ of the energy resolution by a factor of 3 and to have a minimum of 25 counts per bin, to allow the use of the $\chi^{2}$ statistic. We rebinned the RGS spectra to over-sample the FWHM of the energy resolution by a factor of 2 to be sensitive to narrow features and we used the C-statistic. We did not rebin the JEM-X and ISGRI spectra to avoid losing spectral resolution. We performed spectral analysis using XSPEC (Arnaud 1996) version 12.3.1. We used the photo-electric cross-sections of Wilms et al. (2000) to account for absorption by neutral gas with solar abundances (the tbabs XSPEC model). Spectral uncertainties are given at $90 \%$ confidence $\left(\Delta \chi^{2}=2.71\right.$ for one interesting parameter), and upper limits at $95 \%$ confidence.

\subsubsection{EPIC pn and INTEGRAL spectral analysis}

We fit the 0.6-10 keV EPIC pn, 5-25 keV JEM-X and 15-70 keV ISGRI spectra of XB 1254-690 with a model consisting of a disc blackbody and a thermal Comptonisation model, comptt, both modified by photo-electric absorption by neutral and ionised material together with two Gaussian emission features at $\sim 1.0 \mathrm{keV}$ and $\sim 6.4 \mathrm{keV}$ modified by neutral absorption only and one Gaussian absorption feature at $\sim 1.8 \mathrm{keV}$ (tbabs*warmabs*(diskbb+comptt) + tbabs*(gau +gau $)+g a u)$. The feature at $1.8 \mathrm{keV}$ is probably due to an incorrect modeling of the Si absorption in the CCD detectors by the EPIC pn calibration and is therefore not further discussed. Constant factors, fixed to 1 for the EPIC pn spectrum, but allowed to vary for the JEM-X and ISGRI spectra, were included multiplicatively in order to account for cross-calibration 
Table 3. X-ray observations of XB 1254-690 dip states.

\begin{tabular}{lclc}
\hline \hline Date & Mission & Reference & Dip depth \\
\hline 1984 February 5 & EXOSAT & Courvoisier et al. (1986) & Shallow, Undetected \\
1984 May 15 & EXOSAT & Courvoisier et al. (1984) & Shallow \\
1984 August 6-7 & EXOSAT & Courvoisier et al. (1986) & Deep \\
1985 April 14-15 & EXOSAT & Courvoisier et al. (1986) & Undetected, shallow \\
1990 August 1-3 & Ginga & Uno et al. (1997) & Deep \\
1997 April 28 - May 1 & RXTE & Smale \& Wachter (1999) & Undetected, shallow \\
1998 December 22-23 & BeppoS AX & Iaria et al. (2001) & Undetected \\
2001 January 22 & XMM-Newton & Boirin \& Parmar (2003) & Deep \\
2001 May 9-12 & RXTE & Smale et al. (2002) & Deep, undetected, shallow \\
2001 December 6-7 & RXTE & Smale et al. (2002) & Undetected \\
2002 February 7-8 & XMM-Newton & Boirin \& Parmar (2003) & Undetected \\
2003 October 10 & Chandra & Iaria et al. (2007) & Undetected \\
2004 May 26 & RXTE & Barnes et al. (2007) & Deep, undetected \\
\hline
\end{tabular}

Dip states are classified according to the integrated reductions in the X-ray light curves using the definition in Table 1. We have chosen the lowest energy range available for the light curves, where the dips are likely to become more apparent: 1-3.8 keV (EXOSAT), 2-6 keV (Ginga), 2-4 keV (RXTE), 0.7-2.5 keV (BeppoS AX), 0.6-2 keV (XMM-Newton), and 1-8 keV (Chandra).
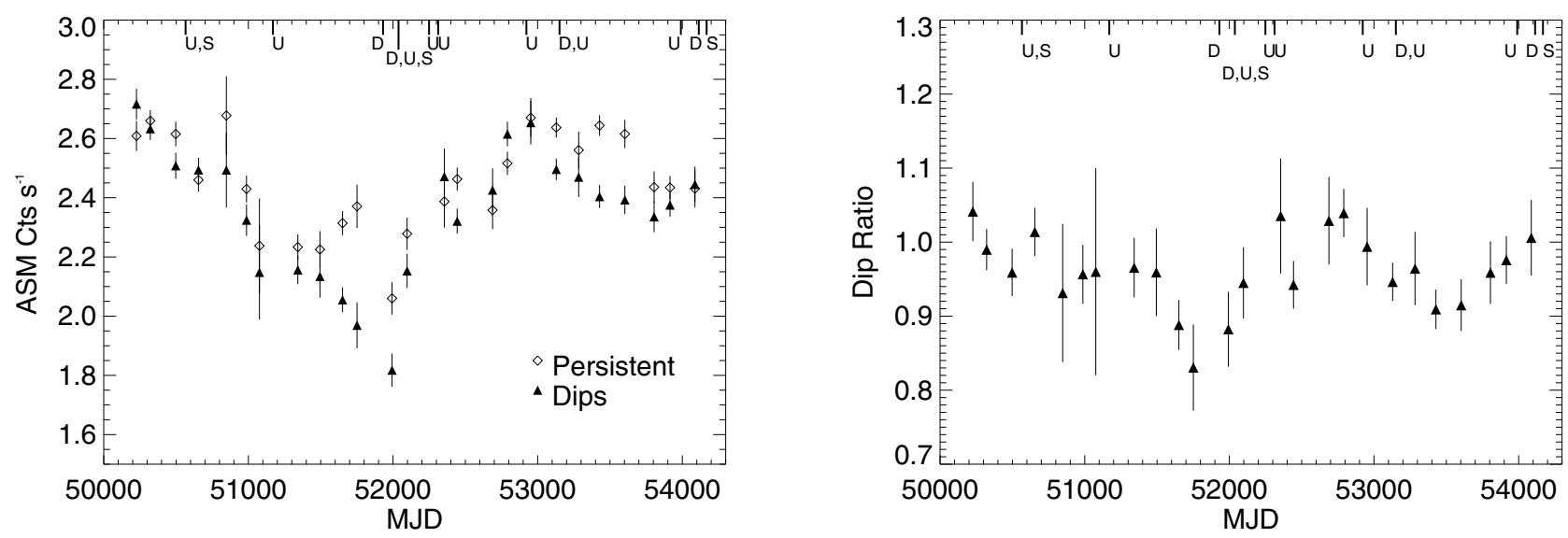

Fig. 8. ASM 1.5-12 keV background subtracted light curve for XB 1254-690 with a binning of 1000 orbital periods or 163.9 days during persistent phase ( $\phi=0.0-0.6$, open diamonds) and dipping phase ( $\phi=0.6-1.0$, filled triangles) (left panel) and the ratio of dipping to persistent phase (right panel). The thick vertical tick marks indicate the dipping state of the source during pointed observations (U: Undetected, S: Shallow, D: Deep; see Table 3). The increase in dip depth around MJD 52000 may be associated with a reduction in persistent count rate.

normalisation uncertainties. The warmabs component models the absorption due to a photo-ionised plasma in the line of sight. All relevant ions are automatically taken into account, including those having small cross-sections, which can contribute significantly to the absorption when combined. The relative column densities of the ions are coupled through a photo-ionisation model. During the fitting process, warmabs calculates spectra using stored level populations pre-calculated with XSTAR (Kallman \& Bautista 2001) which are then scaled using abundances specified during the XSPEC session before the spectra are calculated. A given ionising continuum spectrum is assumed when calculating the level populations with XSTAR (see below for details on the ionising continuum used in this paper). All the models of XSTAR assume a spherical gas cloud with a point source of continuum radiation at the centre. Therefore it implicitly assumes spherical symmetry and radially beamed incident radiation. The main parameters of warmabs are $N_{\mathrm{H}}^{\mathrm{warmabs}}$, $\xi, \sigma_{\mathrm{v}}$, and $v$, representing the column density of the absorber, the ionisation parameter, the turbulent velocity broadening, and the average systematic velocity shift of the absorber (negative values indicate blueshifts). The ionisation parameter is defined as $\xi=L / n R^{2}$, where $L$ is the (energy) luminosity of the incident radiation integrated from 1 to $1000 \mathrm{Ry}, n$ is the gas density, and $R$ is the distance from the radiation source. The warmabs component is necessary to account for the complex residuals evident near $7 \mathrm{keV}$ as well as for modifying the overall continuum shape.

After performing fits for all the observations, it was evident that the disc-blackbody component changes significantly between observations while the thermal Comptonisation component was unchanged within the uncertainties. Therefore, we fit the spectra for all observations together linking the parameters of the thermal Comptonisation, except the normalisation factor, and Gaussian components for all the observations. We kept the normalisation factor of the Comptonisation component unlinked to prevent an artificial variation of the disc-blackbody flux due to the constancy of the former component. When fitting the warmabs component, we first searched for the lowest possible value of the ionisation parameter, $\xi$, which fits the absorption lines well, and then we fixed the parameter to this value to 
Table 4. Best-fits to the 0.6-10 keV EPIC pn, 5-25 keV JEM-X and 15-70 keV ISGRI persistent (non-dip) spectra for all the observations using the tbabs"warmabs* $(\mathrm{dbb}+\mathrm{comptt})+$ tbabs* $\left(\mathrm{gau}_{1}+\mathrm{gau}_{2}\right)$ model.

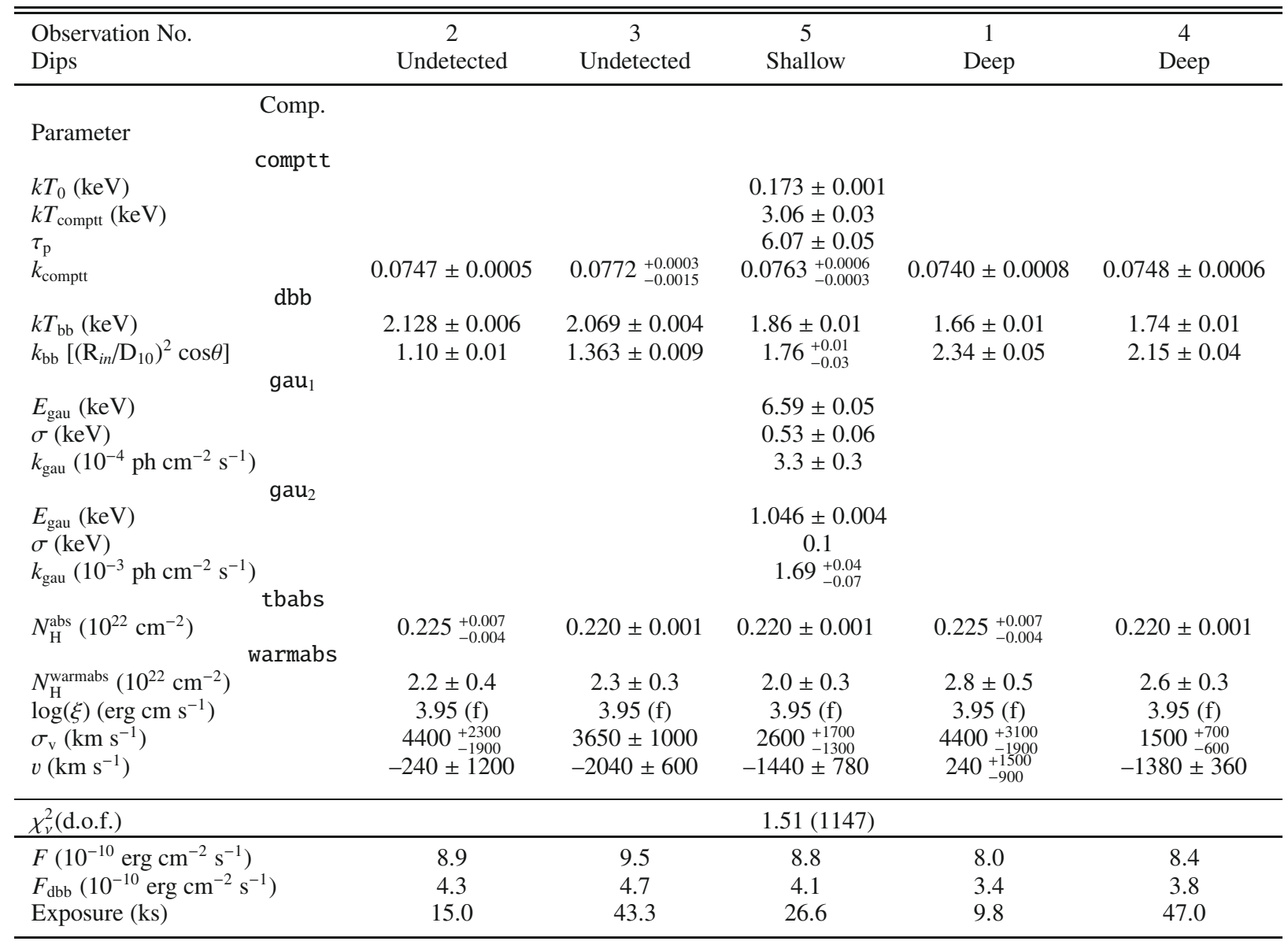

$k_{\text {comptt }}, k_{\mathrm{bb}}$ and $k_{\mathrm{gau}}$ are the normalizations of the Comptonisation component, disc blackbody and Gaussian emission features, respectively. $k T_{0}$ is the input soft photon (Wien) temperature, $k T_{\text {comptt }}$ the plasma temperature, and $\tau_{\mathrm{p}}$ the plasma optical depth, of the Comptonisation component. $k T_{\mathrm{bb}}$ is the temperature of the disc blackbody. $E_{\mathrm{gau}}$ and $\sigma$ represent the energy and width of the Gaussian features. $N_{\mathrm{H}}^{\mathrm{abs}}$ and $N_{\mathrm{H}}^{\mathrm{warmabs}}$ are the column densities for the neutral and ionised absorbers, respectively. $\xi, \sigma_{\mathrm{v}}$, and $v$ are the ionisation parameter (in units of erg $\mathrm{cm} \mathrm{s}^{-1}$ ), the turbulent velocity broadening, and the average systematic velocity shift of the absorber (negative values indicate blueshifts). $F$ is the unabsorbed $0.6-10 \mathrm{keV}$ total flux and $F_{\text {dbb }}$ is the unabsorbed $0.6-10 \mathrm{keV}$ disc blackbody flux. The widths $(\sigma)$ of the Gaussian emission lines gau ${ }_{1-2}$ are constrained to be $\leq 1 \mathrm{keV}$ and $\leq 0.1 \mathrm{keV}$, respectively. $N_{\mathrm{H}}^{\text {abs }}$ is linked for Obs. 1-2 and for Obs. 3-5 during the fits.

obtain the final fits and the uncertainties. This is done to prevent a fit with "artificially" increased continuum normalisations and unrealistic column densities for the absorber.

The $\chi_{v}^{2}$ of the resulting fit is 1.51 for 1147 d.o.f. The parameters of the best-fit model are given in Table 4 and the residuals of the fit are shown in Fig. 9. The values obtained for the neutral absorption are significantly lower than the neutral absorption predicted in the direction of XB 1254-690 by Dickey \& Lockman (1990), $0.31 \times 10^{22} \mathrm{~cm}^{-2}$, and are closer to the value reported in Kalberla et al. (2005) of $0.22 \times 10^{22} \mathrm{~cm}^{-2}$.

Substituting the thermal Comptonisation component by a cutoff power-law component increases the $\chi_{v}^{2}$ of the fit to $\chi_{v}^{2}=2.18$ for 1150 d.o.f. The parameters of the fit remain the same as in Table 4 except for the neutral absorption, which increases to $N_{\mathrm{H}}^{\mathrm{abs}}=(0.307 \pm 0.001) \times 10^{22} \mathrm{~cm}^{-2}($ Obs. 1-2) and $(0.298 \pm 0.001) \times 10^{22} \mathrm{~cm}^{-2}$ (Obs. 3-5). The temperatures (normalisations) of the disc-blackbody component are systematically higher (lower) for all observations compared to the bestfit model, but the relative differences among observations remain the same. The photon index, $\Gamma$, and cutoff energy of the power-law are 2.08 and $34 \mathrm{keV}$, respectively. The value of the energy cutoff is significantly larger than the one obtained from fits to RXTE data by Smale et al. (2002). Substituting the thermal Comptonisation component by a power-law component also increases the $\chi_{v}^{2}$ of the fit to $\chi_{v}^{2}=2.14$ for 1151 d.o.f. The neutral absorption increases to $N_{\mathrm{H}}^{\mathrm{abs}}=(0.321 \pm 0.001) \times 10^{22} \mathrm{~cm}^{-2}$ (Obs. 1-2) and $(0.310 \pm 0.001) \times 10^{22} \mathrm{~cm}^{-2}$ (Obs. 3-5). The index, $\Gamma$, of the power-law is 2.25 .

Substituting the disc-blackbody component by a blackbody component also increases the $\chi_{v}^{2}$ of the fit to $\chi_{v}^{2}=1.90$ for 1133 d.o.f. However, in order to obtain an acceptable fit the thermal Comptonisation component and the neutral absorption must be allowed to vary for each observation.

We used the best-fit Comptonisation component as an ionising continuum for the photoionised absorber. In a previous paper Díaz Trigo et al. (2006) used a different ionisation continuum, consisting of a power law with $\Gamma=1.28$ (obtained from a fit to the Obs. 1 EPIC pn spectrum with only a power law) and a cutoff at $5.9 \mathrm{keV}$ (as obtained from fits to RXTE data by Smale et al. 2002). However, the broad band XMM-Newton 

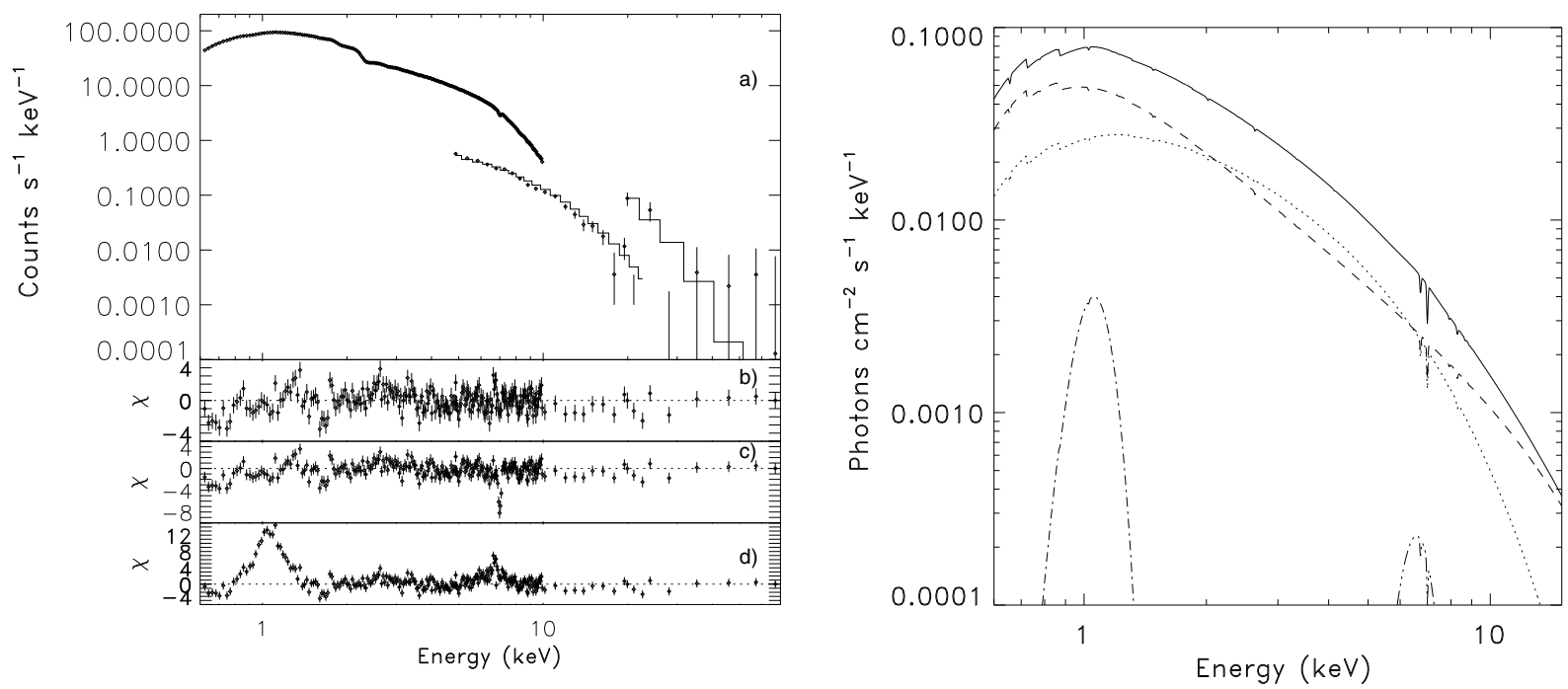

Fig. 9. Left: 0.6-10 keV EPIC pn, 5-25 keV JEM-X and 15-70 keV ISGRI XB 1254-690 Obs. 4 spectra fit with a disc-blackbody (diskbb) and a thermal Comptonisation component (comptt) as continuum modified by absorption from neutral (tbabs) and ionised (warmabs) material together with two Gaussian emission lines $\left(\mathrm{gau}_{1-2}\right)$ which are modified by absorption from neutral material (tbabs) only. b) Residuals in units of standard deviation from the above model. c) Residuals when $N_{\mathrm{H}}^{\mathrm{warmabs}}$ is set to 0 showing the $\sim 7 \mathrm{keV}$ absorption features clearly. d) Residuals when the normalisations of the Gaussian emission lines are set to 0. Best-fit parameters are given in Table 4. Right: best-fit model. Solid, dashed, dotted, dashed-dotted and dashed-dotted-dotted lines represent, respectively, the total model, the comptonisation component, the disc-blackbody component and the Gaussian features at 1 and $6.6 \mathrm{keV}$.

and INTEGRAL spectrum available for Obs. 4 is better fit with a continuum consisting of a disc blackbody and a thermal Comptonisation component than with the model used by Smale et al. (2002). In order to evaluate the influence of the ionisation continuum on our fits, we generated two ionisation continua: one with the two main components of the continuum (disc-blackbody and Comptonisation component) and another one with only the Comptonisation component. The warm absorber is very strongly ionised with only absorption features from H-like Fe XXVI evident. Thus the continuum responsible for ionising the absorber must be the high-energy component, which is in this case the Comptonisation component. Including the disc-blackbody component in the ionising continua did not change the value of the warm absorber ionisation parameter significantly. Thus, we only included the Comptonisation component in the ionising continuum.

\subsubsection{RGS spectral analysis}

We examined the $0.3-2 \mathrm{keV}$ (6-38 $\AA$ ) first and second order RGS spectra. A joint analysis of RGS and pn data was not possible due to the presence of strong emission features in the pn below $3 \mathrm{keV}$ which were not detected in the overlapping energy range of the RGS (see Sect. 2.7.1). We could fit the RGS spectra of all the observations with a continuum consisting of a power law modified by photo-electric absorption from neutral material (C-statistic between 3200 and 4000 for 2820 d.o.f. for Obs. 1 to 5). The RGS spectra show structured residuals near the $\mathrm{O}$ edge at $0.54 \mathrm{keV}$ (see Fig. 10). Around this energy there is a $\sim 25 \%$ change in the instrument efficiency, which may not be fully accounted for in the data processing. Thus, the residuals could be due either to a calibration uncertainties or have an astrophysical origin.

We investigated the second possibility by studying in detail the region around $0.54 \mathrm{keV}(23 \AA)$. We searched for the signature of $\mathrm{O}$ absorption in the interstellar medium similar to that observed by Takei et al. (2002) and Costantini et al. (2005) from Cyg X-2 and by Juett et al. (2004) from a number of galactic sources. We used the tbvarabs absorption model from Wilms et al. (2000) with the oxygen abundance set to zero to allow for an explicit modeling of the oxygen absorption. We included narrow Gaussian absorption features at energies of 0.527 and $0.537 \mathrm{keV}$ (23.5 and $23.1 \AA$, respectively) and an edge at $0.543 \mathrm{keV}(22.8 \AA)$. This improves the fit significantly and we identify the common feature at $0.527 \mathrm{keV}$ with absorption from O I $1 \mathrm{~s}-2 \mathrm{p}$ and the feature at $0.537 \mathrm{keV}$ with absorption from O III $1 \mathrm{~s}-2 \mathrm{p}$. We note that the O II $1 \mathrm{~s}-2 \mathrm{p}$ absorption feature expected at $\sim 0.531 \mathrm{keV}(23.3 \AA)$ falls into an RGS bad column and therefore cannot be detected. In addition, an absorption feature at $0.711 \mathrm{keV}(17.4 \AA)$ is evident in the first and second order RGS spectra of all the observations. We attribute this feature to absorption by the L3 $\left(2 \mathrm{p}_{3 / 2}\right)$ edge of interstellar Fe. Including narrow Gaussian absorption features to model these residuals reduces the C-statistic to 3000-3400 for 2800 d.o.f. for Obs. 1 to 5.

None of the observations show any additional narrow features and including absorption from a photo-ionised plasma in the model does not improve the fit quality. The upper limits to the equivalent widths of highly ionised features such as $\mathrm{Ne} X$ and $\mathrm{Mg}$ XII are $0.35 \mathrm{eV}$ and $0.23 \mathrm{eV}$ (Obs. 3), respectively. This result is consistent with the equivalent widths of the $\mathrm{NeX}$ and $\mathrm{Mg}$ XII, 0.13 and $0.20 \mathrm{eV}$, respectively, predicted for an ionised absorber of $\log \xi=3.95$, as observed in the EPIC pn exposures.

\section{Discussion}

We have analysed five XMM-Newton observations of the LMXB XB 1254-690. The 0.6-10 keV EPIC pn lightcurves are highly variable with two observations showing deep dips, one showing shallow dips and two without evidence of dipping behaviour. We show that the dips identified as "shallow" when plotted with a time resolution of $120 \mathrm{~s}$ include a small number of rapid, deep, variations when plotted with a time resolution of $1 \mathrm{~s}$. The properties of these features appear similar to those observed during deep dipping episodes, where many more such rapid 


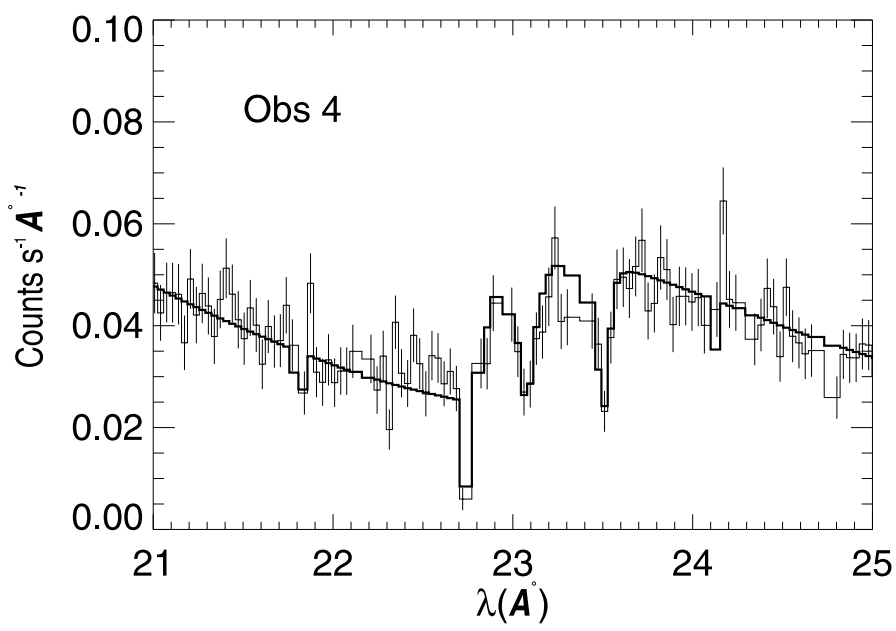

Fig. 10. XB 1254-690 21-25 ̊ RGS spectra for Obs. 4, which has the longest exposure. The structure around the $\mathrm{O}$ edge with clear absorption features at the $1 \mathrm{~s}-2 \mathrm{p}$ transitions of $\mathrm{O}$ I and $\mathrm{O}$ III is shown.

variations can combine, forming the much longer deep dipping intervals observed. The X-ray continua of the dip-free intervals are well fit by a model consisting of a disc-blackbody with a thermal Comptonisation component extending to higher energies. Both components are absorbed by neutral and ionised material. The temperature of the disc blackbody is lower for observations with deep dips $(1.66 \pm 0.01$ and $1.74 \pm 0.01 \mathrm{keV})$, increases for the observations with shallow dips $(1.86 \pm 0.01 \mathrm{keV})$ and is highest for the observations where dips are absent $(2.128 \pm 0.006$ and $2.069 \pm 0.004 \mathrm{keV}$ ). Because the normalisation and temperature of the disc blackbody are highly correlated, it is necessary to examine the confidence contours to evaluate the error range properly. We calculated the confidence contours and found that the range of uncertainty for the temperature is at most $\pm 0.05 \mathrm{keV}$. This means that the change of the temperature is highly significant even if the parameter correlation is taken into account. We did not find any significant changes in the ionisation parameter of the absorber between the persistent emission from the different observations. However, the absorber is very strongly ionised and only features from H-like Fe XXVI are evident, making it more difficult to detect ionisation changes compared to a less ionised plasma where spectral features from a range of ions are visible. In addition, we have taken advantage of the optical monitoring afforded by the OM on XMM-Newton to produce simultaneous folded optical and X-ray light curves of the three long observations which (luckily) cover shallow, deep and dip-free intervals. Finally, we searched for a characteristic dip appearance-disappearance timescale in the available XB 1254-690 X-ray light curves. The RXTE ASM light curves, spanning more than twelve years, are highly suited for detecting long term variability, which in the case of XB 1254-690, we expect to be of the order of a few days. We have explicitly looked for a recurrence period in the appearance of dips in the light curves. Though we find indications of a characteristic timescale of $\sim 60 \mathrm{~h}$ in the RXTE PCA pointed observations, the RXTE ASM light curves are not sensitive enough to confirm such a timescale. Clearly, further observations are necessary. The RXTE ASM curves show however a period with a persistent count rate $\$ 2.2$ cts s$^{-1}$ spanning $\sim 2$ years, which is associated with deep dipping activity. Therefore, there seem to be two processes involved with different timescales which determine the appearance and disappearance of dipping activity.

\subsection{Light curve modelling}

The folded OM light curve for Obs. 3 is similar to those obtained by Smale \& Wachter (1999). We find the same optical average magnitude, $V=19.0$, and amplitude, $\Delta V=0.3$, as in the 1997 optical observations. Both in the 1997 RXTE observation and in Obs. 3, X-ray dips are absent. In Obs. 4, the folded lightcurves are very similar to the February 1984 observations shown in Courvoisier et al. (1986). They report an average magnitude $V=19.0$ and $\Delta V=0.4$ for the optical modulation. However, we extrapolate from their Fig. 3 an average magnitude of $V=19.1$. These values are consistent with those shown in Table 2 for Obs. 4. Finally, whilst the RXTE light curve from Barnes et al. (2007) shows one deep dip, the shape of the simultaneous optical curve seems to be more similar to that of XMM-Newton Obs. 5. Unfortunately, Fig. 3 of Barnes et al. (2007) is in units of flux density rather than magnitude and we cannot easily compare their results with our fits. However, we note that the optical curve from Barnes et al. (2007) covers several cycles while only one X-ray cycle is shown. We have observed that the X-ray behaviour has changed in the cycles following the dip (see Table 3), so this may explain the shape and amplitude of Fig. 3 from Barnes et al. (2007).

Smale \& Wachter (1999) interpret the decrease in optical magnitude and the cessation of dipping activity in the August 1984 to the 1997 observations as an indication of a decrease in the bulge size, implying that the bulge contributes $\sim 35-40 \%$ to the optical modulation. In contrast, Motch et al. (1987) interpreted the shape and phase of the optical modulation as indicating that the majority of the optical modulation is caused by viewing varying aspects of the X-ray heated hemisphere of the companion star with the contribution of the material responsible for the X-ray dips being responsible for $<15 \%$ of the disc emission. The XMM-Newton OM light curves show an optical modulation which is $\sim 20 \%$ of the disc emission, implying that the results of Smale \& Wachter (1999) and Motch et al. (1987) are in disagreement. In the first interpretation, most of the steady component from the optical flux comes from the disc. During dipping intervals, cooler material at the disc rim could obscure part of the hotter, inner disc regions. The disappearance or reduction in size of the azimuthal structure could lead to a more direct view of the hot inner disc regions and an increase in the apparent mean disc emission temperature. This interpretation naturally explains the increase in the temperature of the disc with decreasing dip depth that we observe. However, following this we should observe the $\sim 35-40 \%$ bulge contribution to the optical modulation mainly at $\phi=0.8$, when the X-ray dips are observed, and even a higher contribution at $\phi=0.3$, since the inner bulge should be brighter. Clearly, though we see a significant excess of optical emission at $\phi=0.8$ during deep dipping observations compared to persistent and shallow dipping observations (see Fig. 6), we do not see such excess at $\phi=0.3$, and the fact that the optical minimum is observed at $\phi \sim 0$ is in favour of the optical modulation being caused by the heated hemisphere of the companion.

\subsection{Spectral variability}

We found an apparent blueshift for the Fe XXVI absorption feature in Obs. 3-5. Chandra HETGS observations of the black hole candidates GX 339-4, XTE J1650-500 and H 1743-322 (Miller et al. 2004, 2006b) have revealed the presence of variable, blue-shifted, highly-ionised absorption features which are interpreted as evidence of outflows. In contrast, no blueshifts have been detected in any of the highly-ionised absorbers present 
in dipping LMXBs (Díaz Trigo et al. 2006). However, these results are all obtained with the EPIC pn, which has a factor $\sim 4$ poorer resolution than the HETGS at $6 \mathrm{keV}$ limiting the sensitivity to shifts to be $\gtrsim 1000 \mathrm{~km} \mathrm{~s}^{-1}$. The grating observations of dippers yield strict upper limits for the velocity shifts. We analysed archival data of MXB 1658-298, the only LMXB which shows absorption features of O VIII and Ne X in the RGS (Sidoli et al. 2001), and obtained a velocity shift of $-215_{-270}^{+245} \mathrm{~km} \mathrm{~s}^{-1}$ for the absorber, indicating only a marginal blueshift. Similarly, an upper limit of $\sim 250 \mathrm{~km} \mathrm{~s}^{-1}$ is obtained in Chandra HETGS observations of XB 1916-053 (Juett \& Chakrabarty 2006). The blueshifts found here for XB 1254-690 in Obs. 3-5 seem too large, compared to the maximum shifts of $\sim 400 \mathrm{~km} \mathrm{~s}^{-1}$ obtained elsewhere in LMXBs with a neutron star (see e.g., Ueda et al. 2001) and of $\sim 1000 \mathrm{~km} \mathrm{~s}^{-1}$ in microquasars (see e.g., Miller et al. 2006a). Unfortunately, we do not detect any discrete absorption features from the warm absorber in the RGS, which has a much better energy resolution than EPIC. The lack of spectral features in the RGS wavelength range is consistent with the high degree of ionisation of the absorber. Thus, a more accurate measurement of the blueshifts is not possible for these observations. However, Iaria et al. (2007) report an energy for the Fe XXVI absorption line of $6.962_{-0.015}^{+0.012} \mathrm{keV}$ from Chandra HETGS observations of XB 1254-690, implying that no significant shift is present. Therefore, it seems plausible that the shifts detected with the pn camera do not have an astrophysical origin but are instead due to calibration uncertainties, likely due to an incorrect application of the Charge Transfer Inefficiency (CTI) correction in the EPIC pn camera when high count rates are present ${ }^{2}$. The lines also may be artificially broadened if the inaccuracy of the CTI correction is different for photons arriving at the centre and at the edges of the point spread function.

In addition, a moderately broad $(\sigma=0.5 \mathrm{keV})$ Fe emission line at $6.6 \mathrm{keV}$ with $E W \sim 50 \mathrm{eV}$ and an emission line at $1 \mathrm{keV}$ with $E W \sim 12 \mathrm{eV}$, are present in the spectra. The feature at $1 \mathrm{keV}$ is detected in a number of $\mathrm{X}$-ray binaries and has been previously modelled either as an emission line, or as an edge, and its nature is unclear (e.g., Kuulkers et al. 1997; Sidoli et al. 2001; Boirin \& Parmar 2003; Boirin et al. 2004, 2005; Díaz Trigo et al. 2006). Provided the feature has an astrophysical origin, its appearance always at similar energy points to line emission, since a soft component due to e.g. blackbody emission is expected to change its temperature for different sources. Its energy is consistent with a blend of Ne IX and Ne X emission, or Fe L emission. Recently, broad skewed $\mathrm{Fe} \mathrm{K}$ emission lines have been discovered from a LMXB containing a neutron star (Bhattacharyya \& Strohmayer 2007; Cackett et al. 2008). The emission feature detected in the XB 1254-690 XMM-Newton observations at $6.6 \mathrm{keV}$ is weak and moderately broad. It is not necessary to invoke relativistic effects to explain the moderate line width, which could be due to mechanisms such as Compton broadening. The line is broader and has a larger equivalent width when the disc-blackbody continuum component is substituted by a blackbody. However, the fit quality is worse and the line is unrealistically large. Due to the availability of XMM-Newton and INTEGRAL simultaneous data, we are able to accurately determine the broad band continuum shape and thus we find no evidence for a relativistically broadened Fe line in XB 1254-690.

\footnotetext{
2 More information about the Charge Transfer Inefficiency correction can be found in the EPIC status of calibration and data analysis, by Kirsch et al. (2007), at http://xmm. esac.esa.int
}

\subsection{Explanation for the changes in optical and $X$-ray emission}

We propose that the modulation of the optical light curves is primarily due to obscuration by a precessing accretion disc that is tilted out of the orbital plane. The model is similar to that proposed by Gerend \& Boynton (1976) for Her X-1, and naturally explains the appearance and disappearance of dips observed in the X-ray emission of XB 1254-690, as well as the spectral changes in the continuum seen in different observations. In such a model, the accretion disc performs three major roles in the optical flux variations: the manner in which the disc's X-ray shadow cyclically changes the illumination of the stellar companion, the periodic occultation of the companion by the disc, and the changing optical brightness of the disc itself due to the changing aspect. It is this varying aspect which may cause the changes observed in X-ray luminosity and disc temperature and in addition the periodic appearance and disappearance of dips in XB 1254-690 due to its particular inclination with respect to the observer, $\sim 70^{\circ}$.

A qualitative explanation of the model comes from the schematic precessing disc model shown in Fig. 3 by Gerend \& Boynton (1976). In all cases, the main contribution to the optical flux is due to the X-ray reprocessed emission from the disc, represented in the model that fits the OM light curves by the average magnitude $M$. The angle from which the observer views the pattern of re-emitted light from the disc gradually changes through the precession cycle. A second contribution to the optical flux arises from the heated face of the companion. A maximum contribution of optical light is expected when the observer sees the part of the star facing the disc, $\phi=0.5$, and a minimum when the heated face is out of view, at $\phi=0.0$. This component is represented in our model by the sine component. A secondary minimum in the optical light curve is due to the shadowing by the disc of the heated face at $\phi=0.5$. Depending on the orientation of the disc with respect to the star, such minima could be prominent, as in Obs. 3, or almost invisible, as in Obs. 5. This secondary minimum is also visible in the average light curve in Motch et al. (1987).

In XB 1254-690, dipping should preferentially occur when the inclination of the disc on the line of sight is highest. The disc inclination is never large enough to produce total eclipses, but at orbital phase $\sim 0.8$, the bulge, assumed to be fixed in phase on the disc edge, can partially block the view to the neutron star at some precessional phases. In the case of Her X-1, dipping episodes would correspond to the short "on" state occurring between phases 0.4 and 0.65 in the ephemeris of Gerend \& Boynton (1976). For this particular disc orientation, the $1.7 \mathrm{~d}$ light curve of Her X-1 displays a shoulder at orbital phase $\sim 0.8$ similar to that observed in XB 1254-690. This additional light component is probably due to the X-ray heated stellar region whose centroid is facing the observer, an area unshielded by the disc in this particular geometrical observer-disc configuration. Because of the varying height of the bulge, the appearance of X-ray dips may not necessarily follow a strictly period pattern. This possibly accounts for the fast dip to no dip regime change sometimes observed (e.g. Courvoisier et al. 1986). Non dipping episodes should coincide with low disc inclinations which in the light curve of Her X-1 correspond to precession phases $\sim 0$ (Gerend \& Boynton 1976). At these precessional phases, the disc casts a maximum shadow on the secondary star at inferior conjunction thus producing a secondary minimum at orbital phase 0.5 in Her X-1, similar to that seen in the light curve of Obs. 3. Finally, shallow dipping would occur at an intermediate 
configuraiton between the deep dipping and the non dipping conditions, when the the bulge is observed at grazing angles due to its varying height.

Although disc precession can qualitatively explain the appearance and disappearance of X-ray dips as well as the gross features of the optical light curve, we stress that such an explanation remains unproven in the absence of detailed light curve modelling. In particular, the compatibility of the disc opening angle, bulge height and possible tilt angles with the observed light curve cannot be ascertained.

Finally, an additional effect may be needed to explain the enhanced dipping activity during two years of the ASM light curves.

Acknowledgements. Based on observations obtained with XMM-Newton, an ESA science mission with instruments and contributions directly funded by ESA member states and the USA (NASA) and INTEGRAL, an ESA project with instruments and science data centre funded by ESA member states (especially the PI countries: Denmark, France, Germany, Italy, Switzerland, Spain), Czech Republic and Poland and with the participation of Russia and the USA. This research has made use of data obtained from the High Energy Astrophysics Science Archive Research Center (HEASARC), provided by NASA's Goddard Space Flight Center.

\section{References}

Arnaud, K. A. 1996, in Astronomical Data Analysis Software and Systems V, ASP Conf. Ser., 101, 17

Barnes, A. D., Casares, J., Cornelisse, R., et al. 2007, MNRAS, 380, 1182

Bhattacharyya, S., \& Strohmayer, T. 2007, ApJ, 664, 103B

Boirin, L., \& Parmar, A. N. 2003, A\&A, 407, 1079

Boirin, L., Parmar, A. N., Barret, D., Paltani, S., \& Grindlay, J. E. 2004, A\&A, 418, 1061

Boirin, L., Méndez, M. Díaz Trigo, M., Parmar, A. N., \& Kaastra, J. 2005, A\&A, 436, 195

Cackett, E. M., Miller, J. M., Bhattacharyya, S., et al. 2008, ApJ, 674, 415

Costantini, E., Freyberg, M. J., \& Predehl, P. 2005, A\&A, 444, 187

Courvoisier, T. J.-L., Parmar, A., \& Peacock, A. 1984, in X-ray Astronomy '84, ed. M. Oda, \& R. Giacconi, 153

Courvoisier, T. J. L., Parmar, A. N., Peacock, A., \& Pakull, M. 1986, ApJ, 309, 265
Den Herder, J. W., Brinkman, A. C., Kahn, S. M. et al. 2001, A\&A, 365, L7 Díaz Trigo, M., Parmar, A. N., Boirin, L., Méndez, M., \& Kaastra, J. 2006, A\&A, 445,179

Dickey, J. M., \& Lockman, F. J. 1990, ARA\&A, 28, 215

Gerend, D., \& Boynton, P. E. 1976, ApJ, 209, 562

Griffiths, R. E., Gursky, H., Schwartz, D. A., et al. 1978, Nature, 276, 247

Iaria, R., Di Salvo, T., Burderi, L., \& Robba, N. R. 2001, ApJ, 548, 883

Iaria, R., di Salvo, T., Lavagetto, G., D’Aí, A., \& Robba, N. R. 2007, A\&A, 464, 291

Jansen, F., Lumb, D., Altieri, B., et al. 2001, A\&A, 365, L1

Juett, A., \& Chakrabarty, D. 2006, ApJ, 646, 493

Juett, A. M., Schulz, N. S., \& Chakrabarty, D. 2004, ApJ, 612, 308

Kalberla, P. M. W., Burton, S. B., Hartmann, D., et al. 2005, A\&A, 440, 775

Kallman, T., \& Bautista, M. 2001, ApJS, 133, 221

Kuin, N. P. M., \& Rosen, S. R. 2008, MNRAS, 383, 383

Kuulkers, E., Parmar, A. N., Owens, A., Oosterbroek, T., \& Lammers, U. 1997, A\&A, 323, L29

Labanti, C., Di Cocco, G., Ferro, G., et al. 2003, A\&A, 411, L149

Leahy, D. A., Darbro, W., Elsner, R. F., et al. 1983, ApJ, 266, 160

Lebrun, F., Leray, J. P., Lavocat, P., et al. 2003, A\&A, 411, L141

Lee, J. C., Reynolds, C. S., Remillard, R., et al. 2002, ApJ, 567, 1102

Lund, N., Budtz-Jørgensen, C., Westergaard, N. J., et al. 2003, A\&A, 411, L231

Mason, K. O., Breeveld, A., Much, R., et al. 2001, A\&A, 365, L36

Miller, J. M., Raymond, J., Fabian, A. C., et al. 2004, ApJ, 601, 450

Miller, J., Raymond, J., Fabian, A., et al. 2006a, Nature, 441, 953

Miller, J. M., Raymond, J., Homan, J., et al. 2006b, ApJ, 646, 394

Motch, C., Pedersen, H., Courvoisier, T. J. L., Beuermann, K., \& Pakull, M. W. 1987, ApJ, 313, 792

Scargle, J. D. 1982, ApJ, 263, 835

Sidoli, L., Oosterbroek, T., Parmar, A. N., Lumb, D., \& Erd, C. 2001, A\&A, 379, 540

Smale, A. P., \& Wachter, S. 1999, ApJ, 527, 341

Smale, A. P., Church, M. J., \& Bałucińska-Church, M. 2002, ApJ, 581, 1286

Strüder, L., Briel, U., Dennerl, K., et al. 2001, A\&A, 365, L18

Takei, Y., Fujimoto, R., Mitsuda, K., \& Onaka, T. 2002, ApJ, 581, 307

Turner, M. J. L., Abbey, A., Arnaud, M., et al. 2001, A\&A, 365, L27

Ubertini, P., Lebrun, F., Di Cocco, G., et al. 2003, A\&A, 411, L131

Ueda, Y., Inoue, H., Tanaka, Y., et al. 1998, ApJ, 492, 782

Ueda, Y., Asai, K., Yamaoka, K., Dotani, T., \& Inoue, H. 2001, ApJ, 556, L87

Uno, S., Mitsuda, K., Aoki, T., \& Makino, F. 1997, PASJ, 49, 353

White, N. E., \& Swank, J. H. 1982, ApJ, 253, L61

Wilms, J., Allen, A., \& McCray, R. 2000, ApJ, 542, 914

Winkler, C., Courvoisier, T. J.-L., Di Cocco, G., et al. 2003, A\&A, 411, L1 